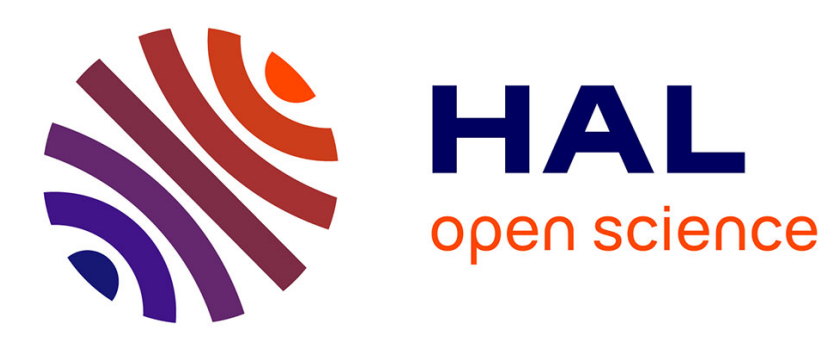

\title{
Improving the power efficiency of SOA-based UWB over fiber systems via pulse shape randomization
}

\author{
H Taki, S Azou, A Hamie, Ali Al Housseini, A Alaeddine, A Sharaiha
}

\section{To cite this version:}

H Taki, S Azou, A Hamie, Ali Al Housseini, A Alaeddine, et al.. Improving the power efficiency of SOA-based UWB over fiber systems via pulse shape randomization. Optical Fiber Technology, 2016, 31, pp.161-167. 10.1016/j.yofte.2016.07.006 . hal-01353969

\section{HAL Id: hal-01353969 \\ https://hal.science/hal-01353969}

Submitted on 17 Aug 2016

HAL is a multi-disciplinary open access archive for the deposit and dissemination of scientific research documents, whether they are published or not. The documents may come from teaching and research institutions in France or abroad, or from public or private research centers.
L'archive ouverte pluridisciplinaire HAL, est destinée au dépôt et à la diffusion de documents scientifiques de niveau recherche, publiés ou non, émanant des établissements d'enseignement et de recherche français ou étrangers, des laboratoires publics ou privés. 


\title{
Improving the Power Eff ciency of SOA-based UWB over Fiber Systems via Pulse Shape Randomization
}

\author{
H. Taki*t, S. Azou ${ }^{\dagger}$, A. Hamie ${ }^{* \ddagger}$, A. Al Housseini*, A. Alaeddine* and A. Sharaiha ${ }^{\dagger}$ \\ * Lebanese University, Faculty of Science I, LPE research center, Beirut, Lebanon \\ ${ }^{\dagger}$ ENIB / CNRS UMR 6285 Lab-STICC, Brest, France \\ ¥CRITC research center, Arts Sciences and Technology University in Lebanon (AUL)
}

\begin{abstract}
A simple pulse shape randomization scheme is considered in this paper for improving the performance of Ultra Wide band (UWB) communication systems using On Off Keying (OOK) or Pulse Position Modulation (PPM) formats. The advantage of the proposed scheme, which can be either employed for Impulse Radio (IR) or for carrier-based systems, is frst theoretically studied based on closed-form derivations of power spectral densities. Then, we investigate an application to an IR-UWB over optical $\mathrm{f}$ ber system, by utilizing the 4-th and 5 -th orders of Gaussian derivatives. Our approach proves to be effective for $1 \mathrm{Gbps}-\mathrm{PPM}$ and $2 \mathrm{Gbps-OOK}$ transmissions, with an advantage in terms of power eff ciency for short distances. We also examine the performance for a system employing an in-line Semiconductor Optical Amplif er (SOA) with the view to achieve a reach extension, while limiting the cost and system complexity.
\end{abstract}

\section{INTRODUCTION}

As there is a growing demand for high speed and low power transmission systems nowadays, ultra wide band (UWB) radio technology becomes an important technique to be used in the new generation of short-range broadband wireless communications [1] [2]. The main advantages offered by UWB are the high data rate, immunity to multipath propagation, f exibility in reconf guring data rate and power, very good time domain resolution, accurate mobile user location, and easy data protection. UWB applications include local and wide area networks, sensor networks, emergency communications, radar, remote sensing, and military applications [3]. Widening UWB system coverage via optical access networks has become an important research topic over the past few years [4]-[8]. Such UWB-over-f ber infrastructures require direct optical pulse generation [9], [10] or electro-optical conversion utilizing intensity modulators [11]. IR-UWB technique has shown a great interest to be used due to its low complexity and cost transceiver architectures [12], [13]. In 2002, the Federal Communication Commission (FCC) allowed UWB devices to operate within a particular spectral mask in order to avoid dangerous interference with other narrow band communication systems [14]. According to this regulation the transmitted power spectral density of UWB has to stay below the limit of $-41.3 \mathrm{dBm} / \mathrm{MHz}$ in the frequency range of $[3.1,10.6] \mathrm{GHz}[15]$. This power limitation has raised the need for highly eff cient IR-UWB signals [16], hence pulse shaping and spectrum smoothing are mandatory to increase the transmitted power without violation of FCC regular limit [17]. A special effort has recently been made by Abraha et al. [18]-[20] for improving the power eff ciency of Gaussian pulses with a lower order of complexity, by using a combination of monocycles and delayed doublets. Pulse shaping can improve the envelope of the overall spectrum, but has no inf uence on the discrete spectral peaks resulting from modulation patterns. Hence smoothing the spectrum of modulated signal requires a change in the statistical properties of the pulse train, time randomization being important so as to reduce the intensity of frequency comb lines. Direct sequence (DS) and Time Hopping (TH) are the mostly utilized randomization techniques, based on varying the pulse amplitude or time position respectively [21]-[23]. The main drawback for DS is the complexity associated with multi-level generation, and randomizing in terms of polarity [24] is not suitable for unipolar encoding like OOK or PPM. Hopping the pulse position between different chips in case of $\mathrm{TH}$ requires a larger symbol duration, which consequently lowers the data rate and may not be pertinent for high speed applications. In this work, a simple randomization technique is adopted for improving the system performance; it consists in changing the pulse waveform over time while keeping the same time location and scaling factor. The energy per bit is conserved for all transmitted frames/symbols, in order to $\mathrm{ft}$ the requirement of non-coherent energy-detection based UWB systems [25]. The proposed Multi-Waveform OOK (MWOOK) and Multi-Waveform PPM (MWPPM) modulation schemes have been applied to a Radio over Fiber (RoF) system. Our scheme has proved a better power eff ciency at short distances of $\mathrm{f}$ ber, which can be extended into several kilometers using a Semiconductor Optical Amplif er (SOA) for in-line amplif cation purpose.

\section{Proposed Multi-WAVEForm OOK/PPM MODULATION FORMATS}

Conventional OOK and PPM impulse radio signals correspond to a stream of modulated pulses via amplitude or position, respectively. The same pulse is systematically repeated along different transmitted frames, leading to a periodicity in time domain and hence to high spectral spikes. Our approach aims to alleviate this undesirable properties by changing the 
pulse shape over time while conserving the digital modulation format principle. Adjusting the pulse waveform is not new in UWB communications [26], but here it is for randomization purpose and not for modulation. In case of an OOK-modulated signal, the proposed Multi-Waveform OOK scheme consists in transmitting different pulse shapes for the bit ' 1 ', each pulse being selected randomly from a particular set $\mathcal{P}$ containing $N$ candidate waveforms, and no transmission is done for bit ' 0 ' as in the conventional scheme. The transmitted signal is then expressed as

$$
w_{M W O O K}(t)=\sum_{k} \sum_{j=1}^{N} b_{j, k} d_{k} p_{j}(t-k T)
$$

where $d_{k} \in\{0,1\}$ denotes the binary data to transmit, $T$ is the frame duration, and $b_{j, k} \in\{0,1\}$ stands for the $j^{\text {th }}$ bit of the $N$-bits coded number $B_{k}=\left(2^{R(k)}\right)_{2}$, with $R(k)$ being a random integer from the uniform distribution on the interval $[0, N-1]$. Hence, $\sum_{j=1}^{N} b_{j, k}=1$ which means that only one pulse $p_{j}$ is triggered in the set $\mathcal{P}$.

Similarly, as illustrated in Fig. 1, a Multi-Waveform PPM can be expressed as

$$
w_{M W P P M}(t)=\sum_{k} \sum_{j=1}^{N} b_{j, k} p_{j}\left(t-k T-d_{k} \Gamma\right)
$$

where $\Gamma$ denotes the modulation index.

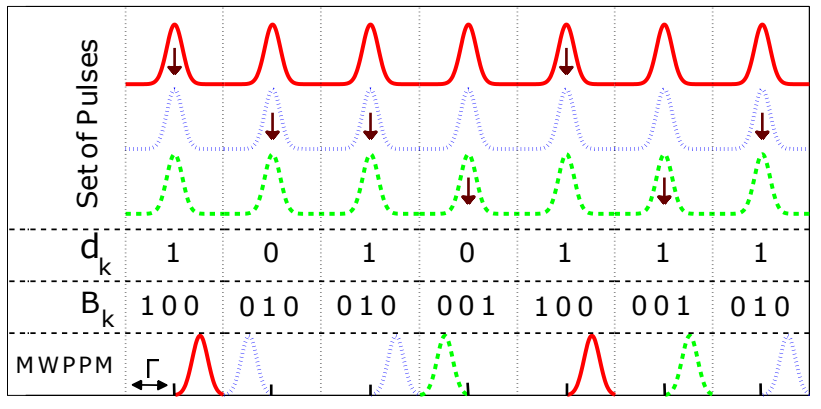

Time Slots and Frames

Fig. 1: A simple description for Multi-Waveform PPM transmission, where each frame consists of 2 time slots.

As different pulses are involved in the randomization process, no correlation must appear between any 2 waveforms from the set $\mathcal{P}$ over the pulse interval $\mathrm{T}$, (notice that frame duration is considered to be equal to the pulse period in this work) :

$$
\int_{0}^{T} p_{m}(t) p_{n}(t) \mathrm{d} t=0 \quad \forall m \neq n
$$

Following a similar approach than that used in [27], closedform expressions of the power spectral densities (PSDs) can be easily derived for the proposed MWOOK/MWPPM formats (see Appendix A for an outline of the proof). For MWOOK, we get the following PSD expression:

$$
\begin{gathered}
S_{M W O O K}(f)=\left|\sum_{j=1}^{N} P_{j}(f)\right|^{2}\left[\frac{\mu_{d}^{2}}{T}\left(\frac{2}{N}-\frac{1}{N^{2}}\right)\right. \\
\left.+\frac{\mu_{d}^{2}}{N^{2} T^{2}} \sum_{k} \delta\left(f-\frac{k}{T}\right)\right]
\end{gathered}
$$

where $\mu_{d}$ stands for the expectation of the binary data $d_{k}$ and $P_{j}(f)$ is the spectrum of pulse $p_{j}(t)$. For the MWPPM format, the PSD takes the form

$$
\begin{aligned}
S_{M W P P M}(f) & =\left|\sum_{j=1}^{N} P_{j}(f)\right|^{2}\left[\frac{1}{T}\left(\frac{1}{N}-\frac{\left|\mu_{m}\right|^{2}}{N^{2}}\right)\right. \\
+ & \left.\frac{\left|\mu_{m}\right|^{2}}{N^{2} T^{2}} \sum_{k} \delta\left(f-\frac{k}{T}\right)\right]
\end{aligned}
$$

where $\mu_{m}=\cos (\pi f \Gamma)$ is the expectation of the random process $m_{k}=e^{-j 2 \pi f \varepsilon_{k}}$ in the spectral domain, with $\varepsilon_{k}=d_{k} \Gamma$.

With the view to characterize the spectrum smoothness, we define the Discrete to Continuous Spectral Ratio (DCSR) as the factor multiplying the series of frequency comb lines divided by the continuous part of the spectrum. For the MWOOK scheme we obtain the following expression

$$
D C S R_{M W O O K}=\frac{1}{T(2 N-1)}
$$

whereas for MWPPM the ratio takes the form

$$
D C S R_{M W P P M}=\frac{\left|\mu_{m}\right|^{2}}{T\left(N-\left|\mu_{m}\right|^{2}\right)}
$$

The DCSR criterion has been established based on theoretical PSD expressions; so, this criterion will no longer be suited to spectral estimates. In the sequel, we will adopt a complementary principle for assessing the benefits of randomization, the Peak-to-Average Spectral Ratio $(P A S R)$, corresponding to be the mean of discrete lines intensity divided by the average of the overall power spectral density.

We will also consider the important criterion of power efficiency, defined as

$$
\eta=\frac{P_{\mathcal{F}}}{\max \left(\overline{P_{\mathcal{F}}}\right)}
$$

where $P_{\mathcal{F}}$ stands for the power collected over a frequency band of interest $\mathcal{F}$ (typically the [3.1-10.6] $\mathrm{GHz}$ band) for the electrical signal at antenna input, and $\max \left(\overline{P_{\mathcal{F}}}\right)$ denoting the total power evaluated over the same band for an OOK or PPM modulated signal based on the sinc pulse, which is optimal in the sense that it corresponds to a $100 \%$ spectrum use (full coverage of the spectral mask).

From Eqs. 6 and 7, it can be clearly seen that increasing the number $N$ of pulses tends to decrease the DCSR, which can result in a better power efficiency as will be illustrated in the next section. Note also that for a unit processing gain 
transmission, the data rate is $R_{b}=1 / T$, so the DCSRs are linear with $R_{b}$. A critical step for taking advantage of the randomized signals is the design of the set of candidate pulses $\mathcal{P}$. One obvious constraint is to conserve energy per bit for all transmitted frames. In addition, we must ensure that the pulses are all mutually uncorrelated (Eq. 3). There are mainly two approaches to achieve this goal, either the transmitter relies on sine and cosine waves with use of local oscillators, or the transmitter directly operates in baseband (Impulse Radio system). The two solutions are investigated in the next section.

\section{CARRIER-BASED VERSUS CARRIERLESS RANDOMIZATION}

\section{A. Carrier-Based Randomization}

To satisfy the decorrelation constraint for a system using local oscillators with inphase and quadrature components, we can design a set $\mathcal{P}$ of rectangular pulses modulated by sine waves at frequencies $f_{n}, n=1,2, \ldots, N / 2$; in this case, the orthogonality is ensured provided that

$$
\int_{-\frac{T}{2}}^{+\frac{T}{2}} \sin \left(\frac{2 \pi f_{i} t}{T}\right) \sin \left(\frac{2 \pi f_{j} t}{T}\right) \mathrm{d} t=0, \forall i \neq j
$$

This is also true in case of sine and cosine waves operating at same frequency. For the purpose of illustration, if we consider a system operating with a pulse period $T$ of $0.5 \mathrm{~ns}$, we can identify 4 frequencies satisfying the orthogonality condition over the [3.1,10.6] $\mathrm{GHz}$ band: $f_{1}=4 \mathrm{GHz}, f_{2}=6 \mathrm{GHz}$, $f_{3}=8 \mathrm{GHz}, f_{4}=10 \mathrm{GHz}$; thus, with 4 local oscillators and using inphase and quadrature components we can get $N=8$ pulses. With this approach, the pulse shape randomization results from a random selection of the sinusoid. As observed in fig. 2 and fig. 3, for both modulation types (MWOOK and MWPPM, respectively), the PASR decreases when increasing the number $N$ of pulses, which means a lower intensity of discrete spectral lines and hence a much more effective randomization. As already pointed out, the frequency comb lines grow linearly with $R_{b}$, so the benefits of using a large set $\mathcal{P}$ is more pronounced at high bit rate but at the price of an increased complexity.

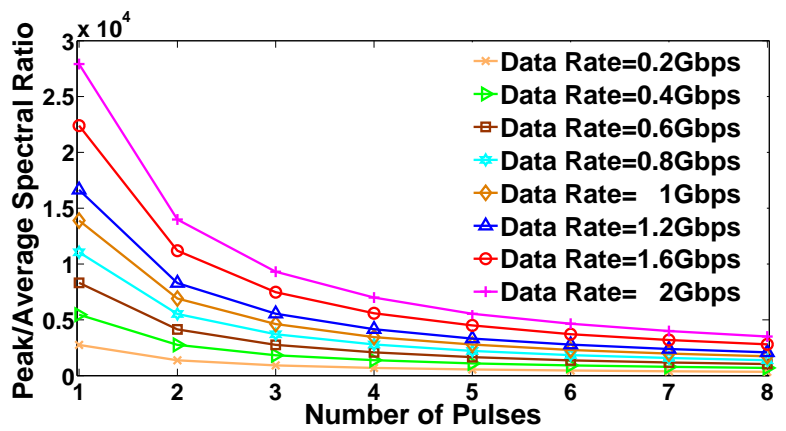

Fig. 2: The Peak-to-Average Spectral Ratio versus number of pulses used for OOK randomization, at different data rates.

The power spectral density for $2 \mathrm{Gbps}$ classical OOK is shown in fig. 4 , besides to the spectrum of multi-waveform

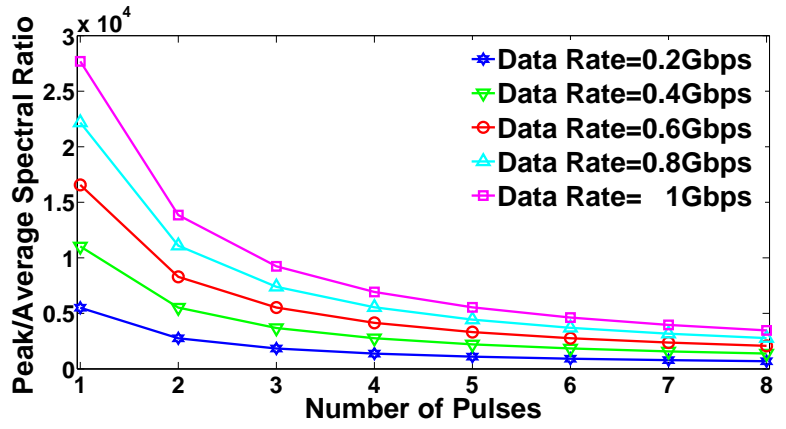

Fig. 3: The Peak-to-Average Spectral Ratio versus number of pulses used for PPM randomization, at different data rates.

one based on 4 pulses. In fig. 5, the spectrum corresponds to 1 Gbps classical and multi-waveform PPM with same $\mathrm{N}$ as in MWOOK (4 pulses). The absence of discrete lines at even multiples of data rate is due to the zero-crossings of sinc spectrum resulting from the $0.5 \mathrm{~ns}$ rectangular pulse. PPM time shift is fixed at $\Gamma=0.5 \mathrm{~ns}$, which is required for non-coherent energy detector receiver. The discrete spectral term is scaled by $\cos ^{2}($.$) as described in (5) associated with spectral peaks$ located at even multiples of data rate, this is a bad spectral phenomenon as comb lines have a higher intensity for PPM than OOK. On the other hand, thanks to the zero crossings of oscillating term which eliminate spectral lines at odd multiples of bit rate, specially at $1 \mathrm{GHz}$ inside GPS band ([0.96-1.61] $\mathrm{GHz}$ ).
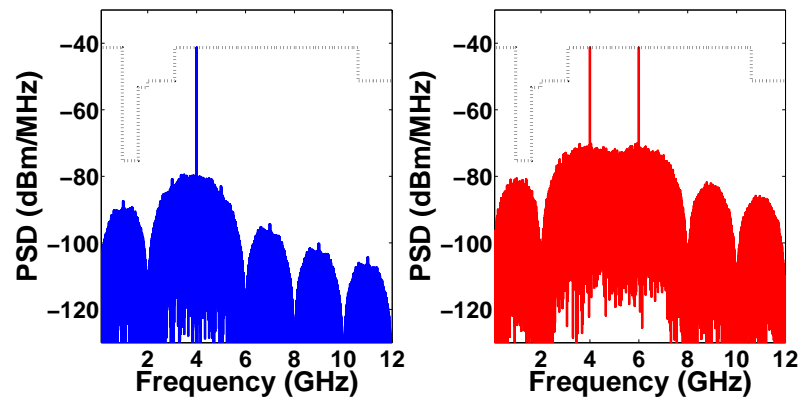

Fig. 4: Power Spectral Densities of 2Gbps standard OOK (left) and multi-waveform OOK based on 4 pulses (right).

As smoothing the power spectral density permits to step-up the spectrum level without violating FCC mask, we can see in fig. 6 a raise in the power efficiency as we increase the number $\mathrm{N}$ of randomizing pulses. Simulations were done for a $1 \mathrm{Gbps}-$ MWPPM and 2Gbps-MWOOK. The lower power efficiency associated with PPM is due to the strong spectral peaks described above. In terms of average power, our randomization scheme shows a consequent improvement with respect to OOK and PPM as observed in fig. 7. That promises with a SNR enhancement when applied in real transmission systems. 

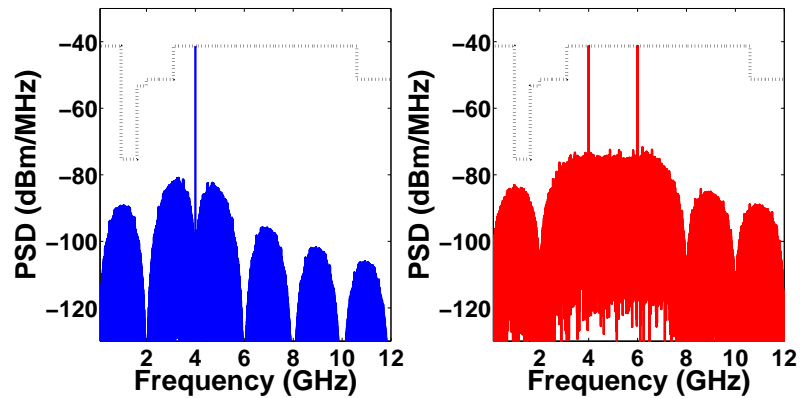

Fig. 5: Power Spectral Densities of 1 Gbps classical PPM (left) and MWPPM based on 4 pulses (right).

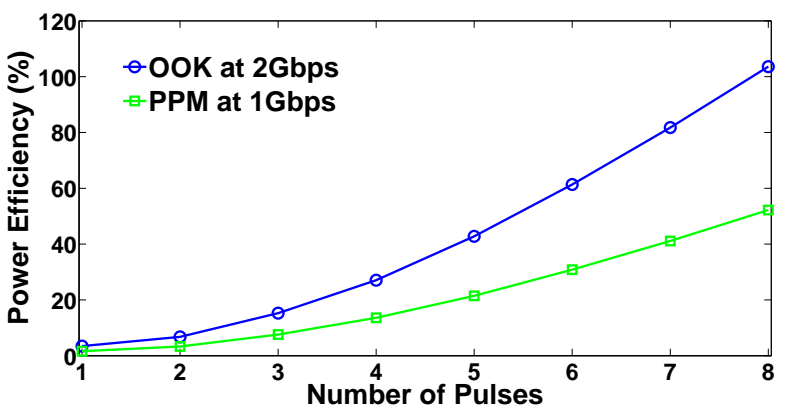

Fig. 6: Power Efficiency versus the number of pulses used for MWOOK and MWPPM.

\section{B. Carrierless Randomization}

The interest of the previous approach is that it gives us some performance bounds for MWOOK and MWPPM schemes, but evidently the use of local oscillator(s) translates into an increase of complexity with respect to a carrierless scheme, which may not be suitable for practical applications. In this section, we investigate the alternate solution of a baseband system (IR-UWB).

With the aim of designing a simple low cost system, we focus on the case where only two pulse waveforms are used for achieving the randomization. There is not a unique choice for the two elementary pulses, but we typically have to respect key constraints such as zero correlation (3), spectral mask compatibility, constant energy, and low complexity of generation. In this study, we considered the 4-th and 5-th order Gaussian monocycles $\left\{g_{4}(t), g_{5}(t)\right\}$, with same pulse shaping factor $\sigma$, as they can easily be generated via CMOS circuits while offering favorable spectral properties [28]:

$$
\begin{gathered}
g_{4}(t)=\left(\frac{t^{4}}{\sigma^{8}}-\frac{6 t^{2}}{\sigma^{6}}+\frac{3}{\sigma^{4}}\right) e^{\frac{-t^{2}}{2 \sigma^{2}}} \\
g_{5}(t)=\left(\frac{-t^{5}}{\sigma^{4}}+\frac{10 t^{3}}{\sigma^{2}}-15 t\right) e^{\frac{-t^{2}}{2 \sigma^{2}}}
\end{gathered}
$$

For the conventional modulation schemes (OOK, PPM), the 5th Gaussian pulse with $\sigma=51$ ps is known to be the optimal choice as it provides the maximum power efficiency [18]. If MWOOK or MWPPM is adopted, a slightly different

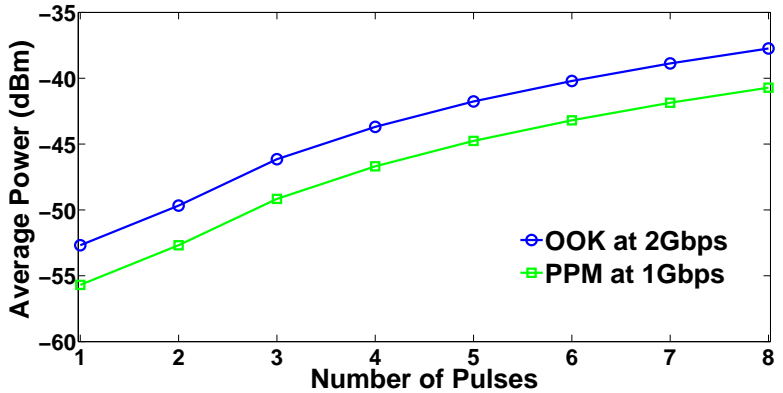

Fig. 7: Average Power versus the number of pulses used for MWOOK and MWPPM.

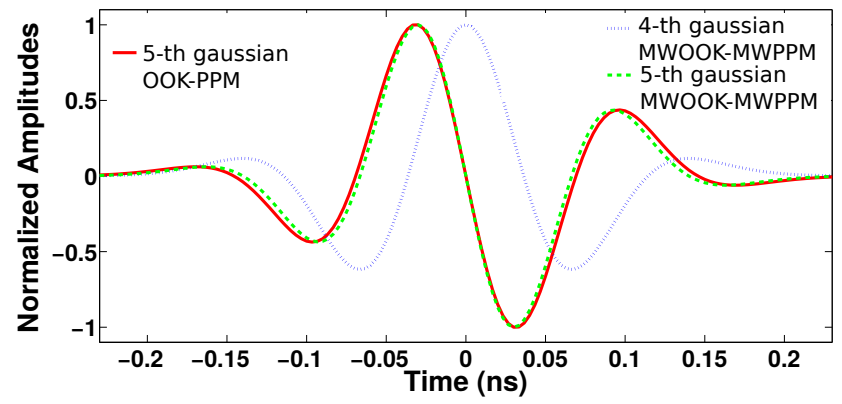

Fig. 8: The 5-th optimal Gaussian used for OOK-PPM, besides to 4 -th/5-th pulses utilized with MWOOK-MWPPM.

shaping factor $\sigma=49$ ps has to be chosen so as to keep a constant energy. Thus, each pulse is no longer optimal with respect to the spectral mask but, as will be shown in the following, favorable power efficiencies will be achieved thanks to the proposed randomizing techniques. Time waveforms and corresponding spectra of utilized pulses are plotted in fig. 8 and fig. 9, respectively. The PASR versus data rate is studied in fig. 10 and fig. 11 for conventional and multi-waveform schemes. Due to the modulation index used for PPM $(\Gamma=0.5$ $\mathrm{ns})$, the maximum rate that can be achieved is 1 Gbps. Since the data rate $R_{b}$ is multiplied by the series of frequency combs (described in Eq. 4 and Eq. 5), we can observe a linear response for both modulation types.

\section{An APPLiCATion to AN IR-UWB OVER Fiber SYSTEM}

Until now, the proposed modulation schemes have been analysed only from a theoretical point of view and their benefits in the context of a realistic channel remains to be investigated. In this section, we consider an application to the Radio-over-Fiber (RoF) depicted in fig. 12. The transmitter relies on a Mach-Zehnder Modulator (MZM) with half-wave voltage $V \pi$ of $6 \mathrm{~V}$, biased at $V_{D C}=1.5 V_{\pi}$ by the electrical IR-UWB signal (peak-to-peak voltage $V_{p p}=0.25 V_{\pi}$ ) with a continuous wave light applied at its input (laser diode signal). The channel is made of a Single Mode Fiber (SMF) with an eventual use of an in-line amplifier (SOA) for the reach extension purpose. At the receiver side (just before wireless 


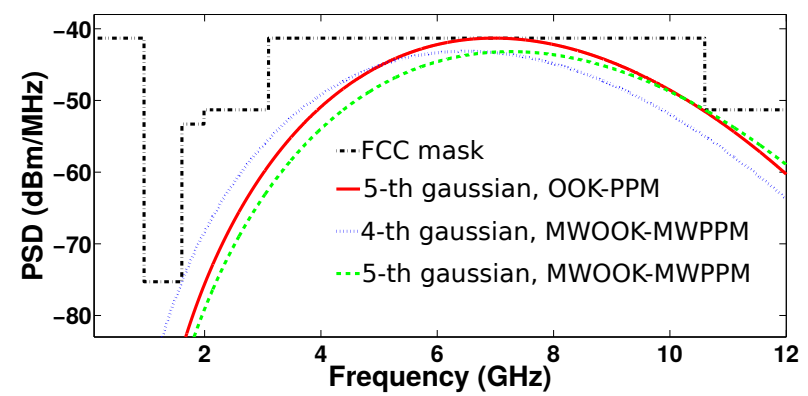

Fig. 9: The power spectral densities of 5-th optimal Gaussian used for standard OOK-PPM, besides to that of 4-th and 5-th pulses utilized with MWOOK-MWPPM.

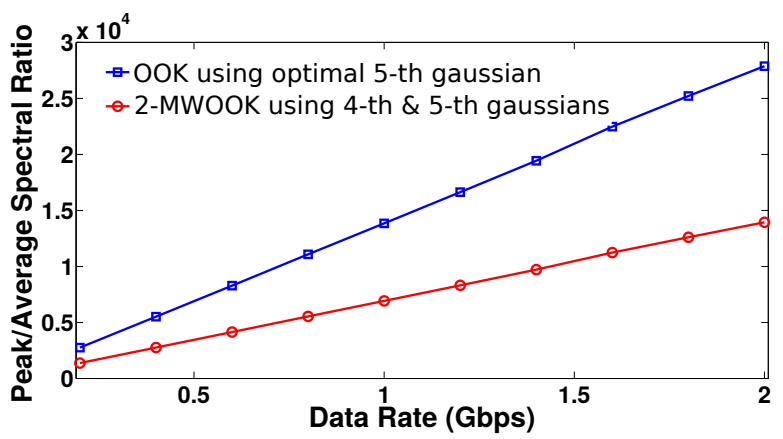

Fig. 10: Peak-to-Average Spectral Ratio versus data rate for OOK-MWOOK based on $4^{\text {th }}$ and $5^{\text {th }}$ derivatives of Gaussian.

transmission), a photo-detector converts the optical power into an electrical voltage signal to be attenuated before entering the antenna (so as to meet FCC mask). The same ADS softwarebased model as in [29] is adopted for the SOA, as it has proved to be highly accurate and in very good agreement with experimental data.

For comparison purpose, we have examined first the performance gain of multi-waveform transmission in the absence of optical amplification. In fig. 13 and fig. 14 we can observe that the power efficiency initially exhibits an almost flat shape over several kilometers whatever the source power $P_{s}$ is. This is due to FCC spectrum violation before attenuator, hence the signal is systematically scaled so as to meet FCC limit. The slight decrease of the power efficiency in this portion of the characteristics, whose width depends upon the source power, is due to the signal attenuation in optical fiber $(0.2$ $\mathrm{dB} / \mathrm{km}$ ). It can be clearly seen that MWOOK and MWPPM schemes offer a significant improvement at short reach, as a result of randomization (lower PASRs). After a certain distance related to $P_{s}$, there is a quick drop-off of the efficiency gain (fig. 14) which then tends towards zero because no spectral lines reduction can be achieved via randomization if the PSD at the attenuator input is already below FCC mask. The efficiency improvement at short reach is larger for MWOOK (close to 9\%) than for MWPPM (slightly above

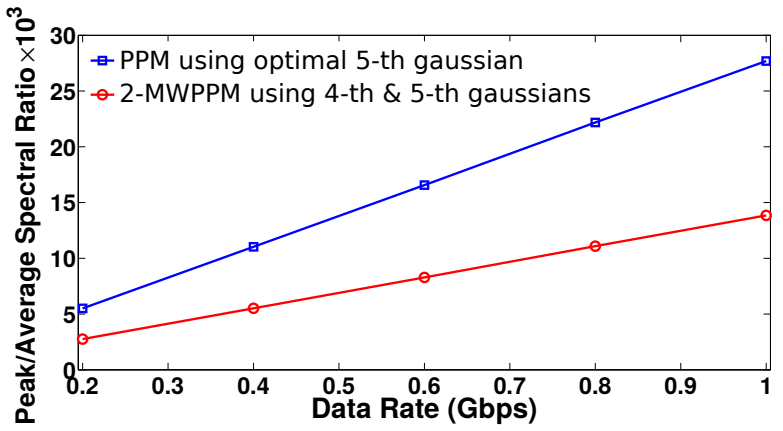

Fig. 11: Peak-to-Average Spectral Ratio versus data rate for PPM-MWPPM based on $4^{t h}$ and $5^{t h}$ derivatives of Gaussian.

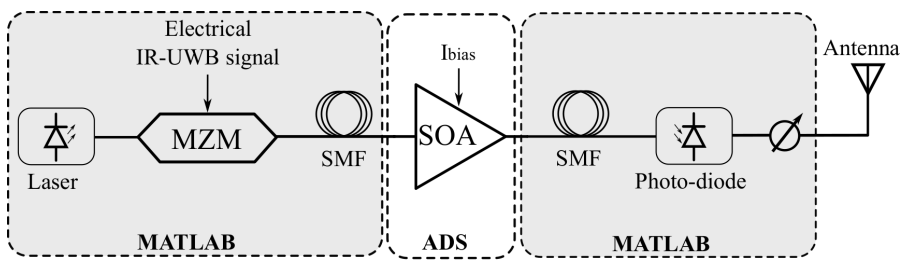

Fig. 12: Block diagram of the Impulse Radio over Fiber system with Matlab/ADS co-simulation.

$4 \%)$. For $P_{s}=12 \mathrm{dBm}$, a performance increase with respect to standard modulation schemes is obtained up to $20 \mathrm{~km}$ and 25 $\mathrm{km}$ for OOK and PPM, respectively. The maximum distance over which the proposed schemes brings an improvement is around $40 \mathrm{~km}$ for a $15 \mathrm{dBm}$ laser power (at this distance the spectrum at the attenuator input falls below the FCC limit). By considering the Fiber-to-the-Home (FTTH) network coverage zones mentioned in the literature [30], $40 \mathrm{~km}$ is not sufficiently large for evaluating the benefits of randomized transmission; thus a SOA was placed as in-line amplifier at this distance to get a reach extension at limited cost. A comparison of the PSDs corresponding to standard modulation schemes and MWOOK/MWPPM, for the whole stream of symbols at SOA output, is illustrated by fig. 15 and fig. 16. Lower PASRs can be clearly observed. For the PPM modulations, the spectrum envelope is influenced by the oscillating term with a period of $2 \mathrm{GHz}$ resulting from the $0.5 \mathrm{~ns}$ shifting in time domain. Power efficiency and corresponding performance gain are plotted in fig. 17 and fig. 18 versus fiber length, considering different values of the biasing current $\left(I_{\text {bias }}\right)$, for a laser power of $15 \mathrm{dBm}$. An evident performance improvement can again be observed for proposed schemes until the distance matches a certain limit related to $I_{\text {bias }}$. Injecting high values of biasing current extends the target distance nonlinearly, a better efficiency being obtained for MWOOK and MWPPM up to $95 \mathrm{~km}$ and $105 \mathrm{~km}$ respectively, when the SOA is biased at $200 \mathrm{~mA}$. The received optical power at SOA input is $-1 \mathrm{dBm}$, boosted by an optical gain of $4.47 \mathrm{~dB}, 10.58 \mathrm{~dB}$, or 11.75 $\mathrm{dB}$; for $I_{\text {bias }}=100 \mathrm{~mA}, 150 \mathrm{~mA}$, or $200 \mathrm{~mA}$, respectively. Another point to mention is that high biasing current tends to slightly lower the performance gain at short distances due 


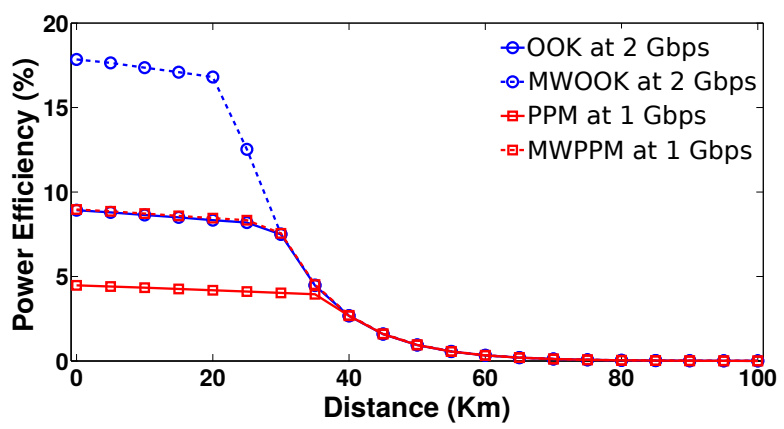

Fig. 13: Power Efficiency versus Fiber Length, where MZM is injected by $15 \mathrm{dBm}$ Laser Power.

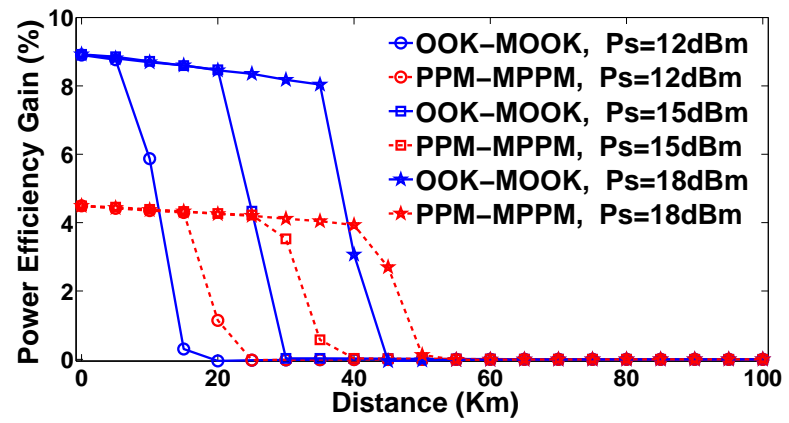

Fig. 14: Power Efficiency Improvement (or Gain) versus Fiber Length for different input powers applied to MZM.

to the nonlinear effects inherent to SOA amplification. So when the spectrum at the photo-detector output is above the FCC limit, linearity of the optical amplifier has a priority over output power response, that is why the best performance gain (close to $10 \%$ ) is obtained when applying $I_{\text {bias }}=100 \mathrm{~mA}$, provided that the receiver is located at a distance below 85 $\mathrm{km}$ (above this limit, MWOOK/MWPPM do not offer any advantage). Operating at larger biasing currents means slightly less power efficiency gain; we then have a trade off between reach extension and power efficiency.

\section{CONClusion}

In this paper, a new spectrum smoothing technique is developed on the basis of OOK or PPM modulation schemes, by applying a pulse shape randomization. The benefits of the approach is first demonstrated by deriving closed-form expressions of the power spectral densities. It is shown that using a greater number of pulses leads to a better spectral behavior, and consequently higher power efficiency. The technique is compatible both with carrier-based transmitters and with impulse radio (IR) systems. With the view to propose a very simple implementation, we investigated the particular case of an IR using two pulses belonging to the gaussian family (4-th and 5-th derivative). Further, we investigated an application to an IR-UWB over optical fiber transmission system operating at $1 \mathrm{Gbps}$ for PPM format and at $2 \mathrm{Gbps}$ for OOK. A significant improvement at short reach of the optical link has been demonstrated if a randomized transmission is
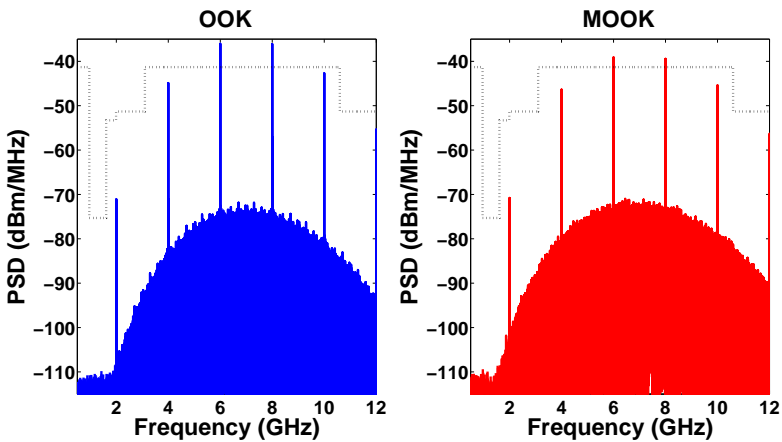

Fig. 15: Power Spectral Densities for 2 Gbps OOK-MWOOK based on $4^{t h}-5^{t h}$ derivatives of Gaussian Pulses at SOA output, where $I_{\text {bias }}=100 \mathrm{~mA}$.
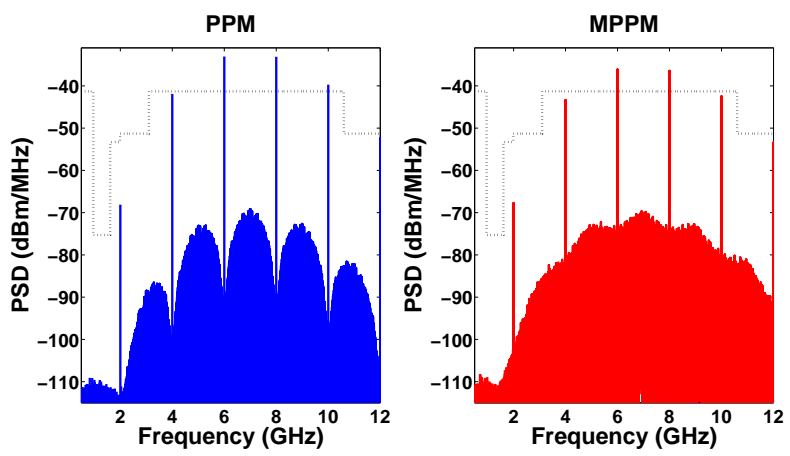

Fig. 16: Power Spectral Densities for 1 Gbps PPM-MWPPM based on $4^{\text {th }}-5^{\text {th }}$ derivatives of Gaussian Pulses at SOA output, where $I_{\text {bias }}=100 \mathrm{~mA}$.

adopted. An eventual in-line optical amplification via SOA is examined for reach extension purpose at low-cost, a realistic model of the component being used. The active distance over which the multi-waveform modulation schemes perform better is controlled by the biasing current injected into the SOA in a flexible way. Considering the antenna and wireless channel is among our future objectives, so as to quantify the benefits of the proposed solutions in terms of Bit-Error-Rate.

\section{APPENDIX}

We give here an outline of the proof of equations (4) and (5), by following similar steps to those used in [27]. Let's first adopt a general expression for the multi-waveform modulation schemes:

$$
w(t)=\sum_{k} \sum_{j=1}^{N} b_{j, k} a_{k} p_{j}\left(t-k T-\varepsilon_{k}\right)
$$

where the amplitude coefficient $a_{k}$ equals the binary data $d_{k}$ in the MWOOK case and equals zero for MWPPM; regarding the time shift, we have $\varepsilon_{k}=0$ for MWOOK and $\varepsilon_{k}=d_{k} \Gamma$ for MWPPM. The transmitted signal can be alternatively expressed as a convolution product:

$$
w(t)=\sum_{j=1}^{N} d_{j}(t) * p_{j}(t)
$$




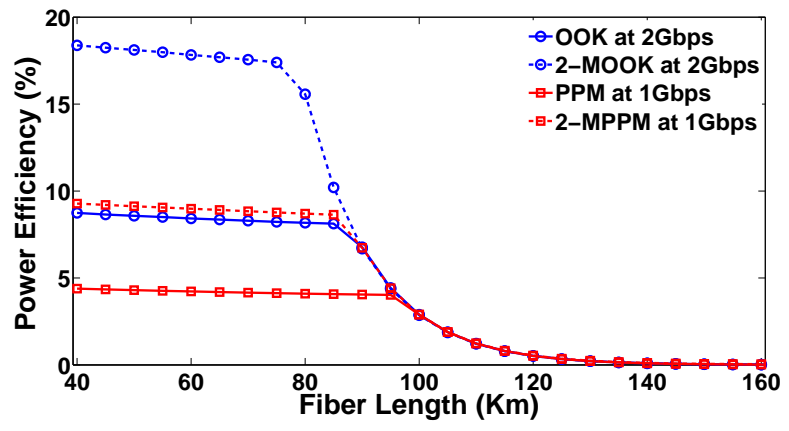

Fig. 17: Power Efficiency versus Fiber Length, using SOA placed at $40 \mathrm{~km}$, and biased with $150 \mathrm{~mA}$ at $15 \mathrm{dBm}$.

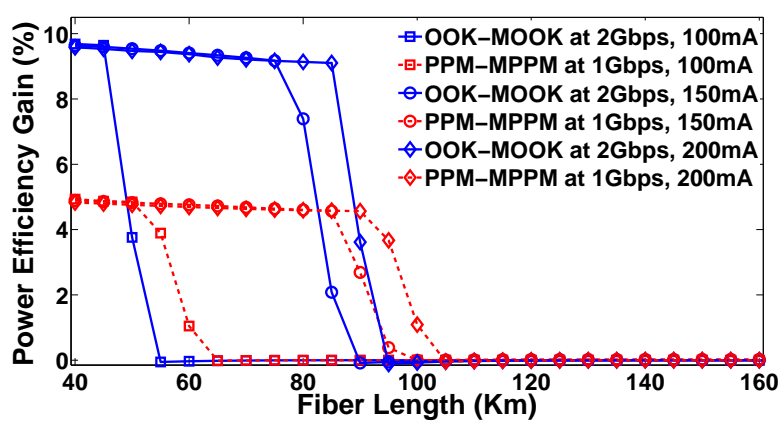

Fig. 18: Power Efficiency Improvement (Gain) versus Fiber Length in case of utilizing SOA at $40 \mathrm{~km}$ for several biasing currents, with a laser power of $15 \mathrm{dBm}$.

where the impulse train signal $d_{j}(t)=$ $\sum_{k} b_{j, k} a_{k} \delta\left(t-k T-\varepsilon_{k}\right) \quad$ reflects the modulation scheme adopted (MWOOK or MWPPM).

Assuming that the set $\mathcal{P}$ is made of mutually uncorrelated pulse waveforms and that the pulse trains $d_{j}(t)$ have the same spectral characteristics, we get the power spectral density

$$
S_{w}(f)=S_{D}(f)\left|\sum_{j=1}^{N} P_{j}(f)\right|^{2}
$$

where $S_{D}(f)$ stands for the PSD of the pulse train and $P_{j}(f)$ being the Fourier transform of $p_{j}(t)$. The discrete spectral lines resulting from the modulation pattern correspond to the term $S_{D}(f)$ only; with the view to derive its expression, it can be written as

$$
S_{D}(f)=<\mathbb{E}\left[\frac{d|D(f, t)|^{2}}{d t}\right]>
$$

where $<.>$ corresponds to time averaging and the accumulated energy spectral density at time $t$ taking the form

$$
|D(f, t)|^{2}=\left|\int_{-\infty}^{t} d(\tau) e^{-j 2 \pi f \tau} d \tau\right|^{2}
$$

After some algebra, it can be seen that the following equations holds:

$$
\begin{gathered}
|D(f, t)|^{2}=\sum_{k} \sum_{l} b_{k} b_{k+l} a_{k} a_{k+l} e^{2 \pi f T l j} e^{2 \pi f j\left(\varepsilon_{k+l}-\varepsilon_{k}\right)} \times \\
u(t-k T) u(t-k T-l T)
\end{gathered}
$$

where $u(t)$ is the unit step function.

Then, it is straightforward to show that

$$
\begin{aligned}
& \mathbb{E}\left[\frac{d|D(f, t)|^{2}}{d t}\right]=\sum_{k} R_{b}(0) R_{a}(0) \mathbb{E}[\delta(t-k T)] \\
& +\sum_{l<0} \sum_{k} R_{b}(l) R_{a}(l) R_{m}(l) e^{j 2 \pi f T l} \mathbb{E}[\delta(t-K T)] \\
+ & \sum_{l>0} \sum_{k} R_{b}(l) R_{a}(l) R_{m}(l) e^{j 2 \pi f T l} \mathbb{E}[\delta(t-K T-l T)]
\end{aligned}
$$

where $R_{b}(l), R_{a}(l)$ and $R_{m}(l)$ denote the auto-correlation functions of the independent processes $b_{k}, a_{k}$ and $m_{k}=$ $e^{-j 2 \pi f \varepsilon_{k}}$, respectively.

Finally, after time averaging and applying the Poisson formula $\sum_{l} e^{j 2 \pi f T l}=\frac{1}{T} \sum_{k} \delta\left(f-\frac{k}{T}\right)$, we get the expression

$$
\begin{gathered}
S_{D}(f)=\frac{1}{T}\left[\left(\sigma_{\hat{b}}^{2} \sigma_{\hat{a}}^{2}+\sigma_{\hat{b}}^{2} \mu_{a}^{2}+\mu_{b}^{2} \sigma_{\hat{a}}^{2}+\mu_{b}^{2} \mu_{a}^{2}-\mu_{b}^{2} \mu_{a}^{2}\left|\mu_{m}\right|^{2}\right)\right. \\
\left.+\frac{\mu_{b}^{2} \mu_{a}^{2}\left|\mu_{m}\right|^{2}}{T} \sum_{k} \delta\left(f-\frac{k}{T}\right)\right]
\end{gathered}
$$

where $\mu_{\times}$corresponds to the mean of the process $\times$and $\sigma$ denoting the standard deviation of the corresponding zero mean process (e.g. $b_{k}=\mu_{b}+\hat{b_{k}}$ with $\mathbb{E}\left[\hat{b_{k}} \hat{b}_{k+l}\right]=\sigma_{\hat{b}}^{2} \delta[l]$ ).

\section{REFERENCES}

[1] M. Ghavami, L. Michael, and R. Kohno, "Ultra wideband signals and systems in communication engineering," John Wiley \& Sons, 2007.

[2] G. R. Aiello and G. D. Rogerson, "Ultra-wideband wireless systems," IEEE Microwave Magazine, 4(2), pp. 36-47, 2003.

[3] I. Oppermann, M. Hämäläinen, and J. Iinatti, "UWB: theory and applications," John Wiley \& Sons, 2005.

[4] C. Lim, A. Nirmalathas, M. Bakaul, P. Gamage, K. L. Lee, Y. Yang, R. Waterhouse, "Fiber-wireless networks and subsystem technologies," IEEE J. Lightw. Technol., 28(4), pp. 390-405, 2010.

[5] F. Zeng, and J. Yao, "An approach to ultrawideband pulse generation and distribution over optical fiber," IEEE Photon. Technol. Letters, 18(7), pp. 823-825, 2006.

[6] S. Pan, and J. Yao, "UWB-over-fiber communications: modulation and transmission," J. Lightw. Technol., 28(16), pp. 2445-2455, 2010.

[7] M. Popov, "The convergence of wired and wireless services delivery in access and home networks," In Optical Fiber Communication Conference (p. OWQ6). Optical Society of America, 2010.

[8] N. J. Gomes, M. Morant, A. Alphones, B. Cabon, J.E. Mitchell, C. Lethien, and S. Iezekiel, "Radio-over-fiber transport for the support of wireless broadband services," J. of Optical Networking, 8(2), pp. 156178, 2009.

[9] F. Zeng, Q. Wang, and J. Yao, "All-optical UWB impulse generation based on cross-phase modulation and frequency discrimination," Electron. Lett., 43(2), pp. 119-121, 2007.

[10] M. Bolea, J. Mora, B. Ortega, and J. Capmany, "Optical UWB pulse generator using an $\mathrm{N}$ tap microwave photonic filter and phase inversion adaptable to different pulse modulation formats," Optics express, 17(7), pp. 5023-5032, 2009.

[11] Y. Yu, J. Dong, X. Li, and X. Zhang, "Ultra-wideband generation based on cascaded Mach-Zehnder modulators," IEEE Photon. Technol. Letters, 23(23), pp. 1754-1756, 2011.

[12] M. Z. Win and R. A. Scholtz, "Impulse radio: How it works," IEEE Communications Lett., 2(2), pp. 36-38, 1998. 
[13] J. R. Fernandes and D. Wentzloff, "Recent advances in IR-UWB transceivers: An overview," In Circuits and Systems (ISCAS), Proceedings of 2010 IEEE International Symposium, pp. 3284-3287, 2010.

[14] H. Sheng, P. Orlik, A. M. Haimovich, Jr. L. J. Cimini, and J. Zhang, "On the spectral and power requirements for ultra-wideband transmission," In Communications, 2003. ICC'03. IEEE International Conference, Vol. 1, pp. 738-742, 2003.

[15] FCC Report and Order. In the Matter of Revision of Part 15 of the Commission's Rules Regarding Ultra-Wideband Transmission Systems. FCC 02-48, Apr. 2002.

[16] V. Yajnanarayana, S. Dwivedi, A. De Angelis, and P. Händel,"Spectral efficient IR-UWB communication design for low complexity transceivers," EURASIP Journal on Wireless Communications and Networking, 2014(1), pp. 1-13, 2014.

[17] Y. P. Nakache and A.F. Molisch, "Spectral shaping of UWB signals for time-hopping impulse radio," IEEE J. Selected Areas in Commun., 24(4), pp. 738-744, 2006.

[18] S.T. Abraha, C. Okonkwo, P. A. Gamage, E. Tangdiongga, and T. Koonen, "Routing of power efficient IR-UWB wireless and wired services for in-building network applications," J. Lightw. Technol., 30(11), pp. 1651-1663, 2012

[19] S. T. Abraha, C. Okonkwo, H. Yang, D. Visani, Y. Shi, H. D. Jung and T. Koonen, "Performance evaluation of IR-UWB in short-range fiber communication using linear combination of monocycles," J. Lightw. Technol., 29(8), pp. 1143-1151, 2011.

[20] S. T. Abraha, C. M. Okonkwo, E. Tangdiongga, and A. M. J. Koonen, "Power-efficient impulse radio ultrawideband pulse generator based on the linear sum of modified doublet pulses," Optics Letters, 36(12), pp. 2363-2365, 2011.

[21] G. M. Maggio, N. Rulkov, and L. Reggiani, "Pseudo-chaotic time hopping for UWB impulse radio," IEEE Trans. Circ. and Syst. I, 48(12), pp. 1424-1435, 2001.

[22] H. Zhang and T. A. Gulliver, "Biorthogonal pulse position modulation for time-hopping multiple access UWB communications," IEEE Trans. Wireless Commun., 4(3), pp. 1154-1162, 2005.

[23] J. Romme and L. Piazzo, "On the power spectral density of time-hopping impulse radio," Ultra Wideband Systems and Technologies-IEEE, pp. 241244, 2002.

[24] A. Pearce, H. Nie, and Z. Chen, "Spectral spike reduction for ultrawideband impulse radio system," Microwave Symposium Digest, IEEE MTT-S International, pp. 1-3, 2012.

[25] S. Cui and F. Xiong, "UWB system based on energy detection of derivatives of the Gaussian pulse," EURASIP J. on Wireless Commun. and Netw., 2011(1), pp. 1-18, 2011.

[26] J. A. Silva and M. L. De Campos, "Spectrally efficient UWB pulse shaping with application in orthogonal PSM," IEEE Trans. on Communications, 55(2), pp. 313-322, 2007.

[27] J. E. Padgett, J. C. Koshy and A. A. Triolo, "Physical-layer modeling of UWB interference effects," Telcordia Technologies Report to DARPA (NETEX Program), Jan. 2003

[28] T. A. Phan, V. Krizhanovskii, S. K. Han, S. G. Lee, H. S. Oh and N. S. Kim, "4.7 pJ/pulse 7th derivative Gaussian pulse generator for impulse radio UWB," In Circuits and Systems, 2007. ISCAS 2007. IEEE International Symposium, pp. 3043-3046, 2007.

[29] H. Khaleghi, P. Morel, A. Sharaiha, and T. Rampone, "Experimental validation of numerical simulations and performance analysis of a coherent optical-OFDM transmission system employing a semiconductor optical amplifier," J. Lightw. Technol., 31(1), pp. 161-170, 2013.

[30] R. Llorente, T. Alves, M. Morant, M. Beltran, J. Perez, A. Cartaxo, and J. Marti, "Ultra-wideband radio signals distribution in FTTH networks," IEEE Photon. Technol. Lett., 20(11), pp. 945-947, 2008. 